http://dx.doi.org/10.22402/j.rdipycs.unam.3.2.2017.142.212-225

\title{
APOYO PSICOLÓGICO A LA FAMILIA: EFECTO EN LA ESTRUCTURA FAMILIAR DE PACIENTES CON HEMOFILIA
}

\author{
Maricela Osorio-Guzmán y Silvina Graña \\ FES Iztacala, UNAM \\ México
}

\begin{abstract}
RESUMEN
El diagnóstico de hemofilia impacta en la familia rompiendo la homeostasis de la misma. Los objetivos del estudio fueron: analizar la estructura familiar de una muestra de padres que tienen un hijo con hemofilia y describir la estructura familiar de las personas que asisten a terapia psicológica y que son apoyados por el programa "Padres Acompañantes". Participaron 39 madres y padres de pacientes pediátricos con hemofilia de diferente tipo y grado clínico, quienes respondieron el cuestionario FACES III. En los índices de cohesión se encontró que $89 \%$ de las familias presentan una estructura semirrelacionada; respecto a la adaptación, $86 \%$ presentó una estructura rígida. Se evidenciaron diferencias en el nivel de adaptación, dependiendo si la familia asiste o no a tratamiento psicológico. De lo anterior se deriva que la estructura familiar se modifica con la irrupción del diagnóstico de hemofilia y genera vulnerabilidad psicológica en este grupo primario; se concluye que el trabajo psicológico promueve un reequilibro saludable, fomentando una mejor calidad de vida a nivel familiar.
\end{abstract}

\section{Palabras Clave:}

hemofilia, familia, cohesión, adaptabilidad, tratamiento psicoterapéutico

\section{PSYCHOLOGICAL SUPPORT TO THE FAMILY: EFFECT ON THE FAMILY STRUCTURE OF PATIENTS WITH HEMOPHILIA}

\begin{abstract}
Hemophilia diagnosis impacts heavily on the family disrupting its homeostasis. The objective of this study is to analyze the family structure in a sample of parents whose child suffers from hemophilia, and to compare the family structure of people attending psychotherapy sessions who are supported by the 'Accompanying Parents' program. Thirty-nine mothers and fathers of pediatric patients with hemophilia of different type and severity participated in the study and answered the FACES III questionnaire. In the cohesion indexes it was found out that 89 per cent of the families present a semi-related structure; concerning their adaptability, whereas 86 per cent show a rigid one. Adaptation level differences were evinced depending on whether or not families undergo psychological treatment. The sudden diagnosis of hemophilia generates psychological vulnerability in the family group. Psychological therapy promotes a healthy restructuring that fosters better familial quality of life.
\end{abstract}

Keywords:

hemophilia, Family, Cohesion, Adaptability, Psychotherapy treatment

Bitácora del Artículo:

| Recibido: 1 de Agosto de 2017 | Aceptado: 1 Septiembre de 2017 | Publicado en línea: Julio-Diciembre de 2017 | 


\title{
APOYO PSICOLÓGICO A LA FAMILIA: EFECTO EN LA ESTRUCTURA FAMILIAR DE PACIENTES CON HEMOFILIA
}

\author{
Maricela Osorio-Guzmán y Silvina Graña Vitale \\ FES Iztacala, UNAM \\ México
}

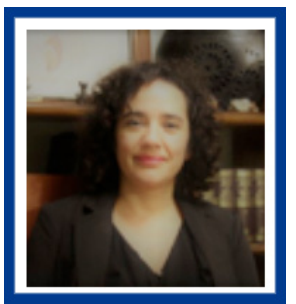

Maricela Osorio-Guzmán

FES Iztacala, UNAM

Correo: mosorio@unam.mx

Profesora titular "C" de tiempo completo de la carrera de Psicología, UNAM, FESI. Doctorado en Ciencias Psicológicas y Pedagógicas, Área: Psicología de la Salud y Prevención del Riesgo Individual y Social por la Universidad de los Estudios de Nápoles Federico II en Italia.

Ver más..

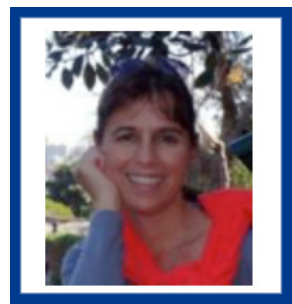

Silvina Graña Vitale FES Iztacala, UNAM Correo: silvinagrana@yahoo.com

Psicóloga clínica. Trabaja en la Fundación de la Hemofilia de la Argentina, con niños, adolescentes y familia. Coordina talleres y encuentros en todo el país. Miembro del Comité Psicosocial de la Federación Mundial de Hemofilia (FMH). Miembro del Comité Multidisciplinario de la FMH para el Congreso Mundial 2018.

Ver más...

\section{CONTRIBUCIÓN DE LAS AUTORAS}

Maricela Osorio-Guzmán concibió la idea, elaboración marco teórico, método, análisis y redacción de datos, conclusiones y referencias. I Silvina Graña estuvo a cargo de la aplicación de los instrumentos, colaboración en el MT, elaboración resumen, abstract y colaboración en la elaboración de conclusiones.

\section{AgRADECIMIENTOS}

Las autoras agradecen a la World Federation of Hemophilia la beca CIEHEMO otorgada para la realización de Capacitación Especializada en el Centro "Dr. Alfredo Pavlovsky" de la Fundación de la Hemofilia e Instituto de Investigaciones Hematológicas "Dr. Mariano R. Castex" (IIHEMA) de la Academia Nacional de Medicina, Buenos Aires, Argentina. A su vez se agradece a la Dra. Daniela Neme, Directora Médica de la Institución, por el apoyo para la realización de esta investigación con la que día a día renovamos el deseo de trabajar en forma muldisciplinaria. Al grupo de Padres Acompañantes, por su generosidad y dedicación.

\section{Datos de FiLiación de las Autoras}

Facultad de Estudios Superiores Iztacala, UNAM. | Fundación de la Hemofilia de la Argentina.

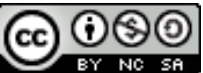

Copyright: (c) 2017 Osorio-Guzmán, M., \& Graña, S.

Este es un artículo de acceso abierto distribuido bajo los términos de la licencia Creative Commons Reconocimiento-NoComercial-Compartirlgual 4.0 Internacional, por lo que su contenido gráfico y escrito se puede compartir, copiar y redistribuir total o parcialmente sin necesidad de permiso expreso de sus autoras con la única condición de que no se puede usar con fines directamente comerciales y los términos legales de cualquier trabajo derivado deben ser los mismos que se expresan en la presente declaración. La única condición es que se cite la fuente con referencia a la Revista Digital Internacional de Psicología y Ciencia Social y a sus autoras. 


\section{TABLA DE CONTENIDO}

INTRODUCCIÓN

MÉTOdo

Participantes, 25

Materiales, 25

Procedimiento, 25

Análisis estadísticos, 25

RESULTADOS

Características demográficas de la muestra, 26

Estructura familiar, correlaciones y diferencias, 26

CONCLUSIONES 


\section{INTRODUCCIÓN}

\section{Trean} a hemofilia es una enfermedad en la sangre que se caracteriza por la deficiencia de uno de los factores de coagulación (Pruthi, 2005). Es una enfermedad congénita; se dice que la transmisión está relacionada con el sexo, ya que el gen responsable se encuentra en el cromosoma $X$; en general las mujeres son portadoras de la enfermedad y los varones son los afectados. Las personas con bajos niveles de factor VIII presentan hemofilia $\mathrm{A}(\mathrm{HA})$, y las que presentan bajos niveles de factor IX, hemofilia B (HB). Ambos tipos de hemofilia son clínicamente iguales, y pueden ser leves, moderados o severos de acuerdo con el nivel de factor circulante. El tratamiento consiste en la aplicación por vía endovenosa del factor deficitario. La incidencia de la hemofilia en la población mundial se estima en 1 de cada 10,000 varones nacidos vivos. La hemofilia se distribuye de manera uniforme en la población, y la Federación Mundial de Hemofilia (FMH) estima que a nivel mundial hay 400,000 personas con esta coagulopatía. Específicamente en la Argentina, hay casi 3,000 casos registrados (Consenso de Médicos Especialistas en Hemofilia de la República Argentina, 2015).

El momento del diagnóstico en un niño con una enfermedad crónica como la hemofilia, es vivido por la mayoría de las familias como una situación traumática, registrándose la aparición de altos niveles de estrés y sufrimiento psicológico. Esto se debe en parte al cambio de la rutina general y a la pérdida de control de la situación familiar por los padres, así como a la incertidumbre derivada de la enfermedad misma (Arranz, Costa, Bayés, Cancio, Magallón y Fernández, 2000; Goldstein, Golan, Kenet y Martinowitz, 2000; Kelley, 2006; Graña y Rodríguez, 2000; Osorio, 2016).

Esta situación produce una crisis dentro del núcleo familiar, rompiendo el equilibrio con que la familia se manejaba hasta ese momento. Esta pérdida de la homeostasis se manifiesta en múltiples niveles como en el ámbito laboral, la relación de pareja, la relación entre hermanos, en el área psicoemocional, etcétera, y diferentes trabajos internacionales dan cuenta de ello (Balcells y Ribalta, 2000; O'Mahony, 2002; Herrick, Nussbaum, Holtzman y Wissow, 2004; Hashim, 2008; Benoit de García, 2008; Goldstein, 2008; Graña, 2010).

Desde el ámbito de la psicología, es relevante realizar entrevistas con los padres para orientarlos y dar seguimiento a sus procesos de afrontamiento ante este nuevo reto. Ade- más, es importante que el espacio se amplíe a toda la familia, incluyendo a los niños con hemofilia, a los hermanos sin la patología, a los maestros y a las personas significativas para este grupo primario (Spilsbury, 2004; Graña, 2014).

En la Fundación de la Hemofilia en Buenos Aires, el consultorio de psicología implementó el programa "Padres Acompañantes", en el cual varios matrimonios, madres y/o padres con niños con hemofilia severa fueron convocados y entrenados por la psicóloga para conformar un grupo de voluntarios; dicho programa oferta un espacio de encuentro con "Padres Nuevos" (diagnóstico reciente de sus hijos) y/o en situaciones de crisis. Los integrantes, por haber ya tenido experiencia con la enfermedad, pueden compartir sus vivencias para mostrar otra manera de relacionarse y convivir con la hemofilia de los hijos y en su familia, es decir, los padres acompañantes funcionan como un otro semejante, como un modelo posible de identificación (Graña, 2010). Además, siguiendo los conceptos de Winnicott (1979), este grupo se ubica en un lugar tercero, funcionando como sostén.

Los objetivos del programa consisten en crear una red de soporte social, familiar e institucional, colaborar en el proceso de aceptación del diagnóstico y en el restablecimiento de la homeostasis familiar, considerando a la hemofilia como una situación más de la vida cotidiana (Graña, 2010).

Por su parte, el abordaje psicológico consiste en realizar entrevistas a las familias de reciente diagnóstico, abordando el impacto de la enfermedad crónica en la familia. Se recibe al grupo familiar primario y, de acuerdo con cada caso, se implementa una serie de sesiones de orientación y seguimiento (Graña, 2010, 2014). De ser necesario, se propone un espacio de psicoterapia que puede ser — como ya se mencionó- familiar, de pareja, a los hermanos sin la patología, a los niños con hemofilia, e incluso a la familia extendida o cuidadores. En este marco también se ofrece la posibilidad de participar en el programa "Padres Acompañantes", teniendo una familia/madre/padre acompañante.

Mientras que en el caso de los adultos la vía privilegiada del trabajo terapéutico es por medio de la palabra, con los niños el trabajo se realiza sobre todo mediante el dibujo y el juego. De acuerdo con Winnicott (1982), lo universal en el niño es el juego; esta actividad es una experiencia creativa, facilitadora de un crecimiento saludable y posibilita las relaciones de grupo. Es principalmente por medio del juego que el niño descubre el mundo, establece relaciones con los otros, haciendo posible la socialización. Asimismo, el juego es el vehículo por el cual los niños expresan sus emociones, ansiedades y preocupaciones. Además, el juego simbólico es la vía regia por la cual los niños elaboran conflictos y situaciones traumáticas. 
La función del psicólogo no es la de mero observador del juego del niño, sino que tiene un rol activo en el mismo, fomentando e interviniendo activamente cuando éste se ve interrumpido o inhibido, para que pueda ser retomado. Además, puede él mismo ser un objeto durante su desarrollo o un personaje en el juego que despliega el infante (Graña, 2010).

Por otra parte, en el área académica y profesional, uno de los modelo que ha tenido una gran difusión y credibilidad en los últimos años es el Modelo Circumplejo de Sistemas Familiares y Maritales (Olson, 2000; Olson, Russell y Sprenkle, 1989; Olson, Sprenkle y Russell, 1979); dicho modelo ha tenido consenso entre los investigadores respecto a la relevancia de dos dimensiones que se consideran críticas para entender y tratar familias que se encuentran bajo niveles altos de estrés, las cuales son la cohesión y la adaptabilidad. Olson, Portner y Lavee (1985) definen cohesión familiar como "el grado de unión emocional (o apego) percibido por los miembros de la familia; mientras que la adaptabilidad (o flexibilidad) está referida al grado en que la familia es capaz de cambiar su estructura de poder, sus roles y reglas en respuesta al estrés (Schmidt, 2001: 31).

Olson, Portner y Lavee (1985, apud Schmidt, 2003), consideran que la cohesión y la adaptabilidad están curvilíneamente relacionadas con la salud familiar, y que las familias que funcionan moderadamente a lo largo de las dimensiones de cohesión y adaptabilidad se conducen de manera más saludable y tienen mayor probabilidad de un afrontamiento eficaz frente a las demandas ambientales, comparadas con familias localizadas en los extremos.

También Schmidt, Barreyro y Maglio (2009) mencionan que el grado de cohesión y flexibilidad que presenta cada familia constituye un indicador del tipo de funcionamiento que predomina en el sistema: extremo, de rango medio o balanceado. Los sistemas maritales o familiares balanceados tienden a ser más funcionales y facilitadores del funcionamiento, siendo más problemáticos los extremos.

De lo expuesto se desprenden los objetivos del presente trabajo, los cuales fueron analizar la estructura familiar de una muestra de padres que tienen un hijo con hemofilia y describir la estructura familiar de las personas que asisten a terapia psicológica y que son apoyados por el programa "Padres Acompañantes".

\section{MÉtodo}

\section{Participantes}

Participaron 39 padres y madres de pacientes pediátricos con hemofilia de diferente tipo y grado clínico, los cuales cumplieron con los siguientes criterios de inclusión: ser padre o madre de por lo menos un niño con hemofilia, saber leer y escribir, y aceptar participar en la investigación. A todos los participantes se les explicó en qué consistía su participación y todos firmaron el consentimiento informado. El proyecto general del cual se desprende esta investigación fue revisado y aprobado por el Comité de Bioética de la Carrera de Psicología de la Facultad de Estudios Superiores Iztacala.

Criterios de exclusión: padres que no supieran leer y escribir o que se rehusaron a participar en la investigación.

El tipo de muestreo utilizado fue intencional o de conveniencia: se caracteriza porque el investigador selecciona directa e intencionadamente los individuos de la población, ya que tienen las características que él desea estudiar; además, las muestras son seleccionadas porque son accesibles para el investigador (Shaughnessy, Zechmeister y Zechmeister, 2007).

\section{Materiales}

Se aplicó un cuestionario diseñado ad hoc para obtener datos demográficos y del padecimiento (éste incluía preguntas como escolaridad, tipo de hemofilia, grado clínico, número eventos hemorrágicos, tipo de tratamiento, número de hospitalizaciones y antecedentes familiares, entre otras); y el FACES III (FamilyAdaptability and CohesionEvaluationScales) en español (Ponce, Gómez, Irigoyen, Terán, Landgrave, Hernández et al., 1999; Ponce et al., 2002), el cual contiene 20 ítems (10 para evaluar cohesión familiar y 10 para adaptabilidad familiar), cuya escala de respuesta es tipo Likert. Los ítems están distribuidos en forma alterna, los reactivos nones evalúan cohesión familiar y los pares adaptabilidad. Los valores van de 1 a 5 (nunca, 1 ; casi nunca, 2; algunas veces, 3; casi siempre, 4, y siempre, 5). El cuestionario tiene una confiabilidad de $\square=0.69$.

\section{Procedimiento}

Se elaboró un cuestionario para obtener datos demográficos e información acerca del padecimiento; se seleccionó el instrumento FACES III; los instrumentos se aplicaron en el consultorio de psicología de la Fundación de la Hemofilia en Buenos Aires, en las fechas y horarios en que pacientes y familiares asistieron al servicio de hematología para los controles semanales/mensuales, o a consulta psicológica. El tiempo aproximado en cada aplicación fue de 15 minutos.

\section{Análisis estadísticos}

Se realizó un análisis descriptivo de las variables sociodemográficas de la muestra; se calcularon y describieron los puntajes de cada una de las escalas que componen el instrumento; se compararon las medias obtenidas en cada área, 
por medio de la t de Student, considerando las variables tipo de hemofilia, severidad, tratamiento psicológico, acompañamiento de padres, etcétera, y se obtuvieron las correlaciones por medio de la prueba $r$ de Pearson entre las diferentes variables y las áreas del cuestionario. Para apoyar el análisis de datos se utilizó el programa estadístico SPSS 22.

\section{Resultados}

\section{Características demográficas de la muestra}

Participaron 39 padres y madres de pacientes pediátricos con hemofilia (33\% hombres y $67 \%$ mujeres), cuya media de edad fue de 38.84 (desviación típica [DT] = 9.76) y las edades fluctuaron de 17 a 64 años. De esta muestra, 14\% pertenece al grupo de padres acompañantes, y $60 \%$ asiste regularmente a atención psicológica. Respecto a su escolaridad, 28\% tiene educación terciaria y $25 \%$ es universitario.

La edad promedio de los pacientes fue de 7.02 años (DT $=4.17$ ); el tipo de hemofilia que padecían los niños era $91 \%$ hemofilia A y el restante $9 \%$ hemofilia B. Respecto al grado clínico, $7 \%$ fue leve, $4 \%$ moderado y $89 \%$ severo. La media de edad del diagnóstico fue de 8 meses (DT $=6.3$ ) y los datos variaron de 0 a 24 meses. Todos los pacientes son tratados con factor, $91 \%$ está en profilaxis y el restante $9 \%$ en tratamiento a demanda. El $67 \%$ no ha tenido ninguna hospitalización, la media es igual a 1.26 hospitalizaciones al semestre (DT $=2.15)$ y $28 \%$ ha tenido uno o dos eventos hemorrágicos en un año (sangrado de tobillo, codo o rodilla). El 36\% tiene un hermano, 18\% dos hermanos, y el rango es de 1 a 4 hermanos. El 51\% de los pacientes con hemofilia $(\mathrm{PCH})$ tienen antecedentes familiares, siendo abuelos y primos los más comunes.

\section{Estructura familiar, correlaciones y diferencias}

Respecto a los promedios de los índices obtenidos en la prueba Faces III aplicada, es interesante observar la variabilidad de los datos mínimos y máximos en las escalas de cohesión - la cual, como ya se dijo, es el grado de unión emocional (o apego) percibido por los miembros de la familia - y adaptabilidad - la que hace referencia al grado en que la familia es capaz de cambiar su estructura de poder, sus roles y reglas en respuesta al estrés- $(\mathrm{DE}=5.7$ y 5.2, respectivamente) (tabla 1$)$.

Tabla 1.

Índices de dispersión de las áreas del Faces III.

\begin{tabular}{cccccc} 
Área & Media & $\begin{array}{c}\text { Desviación } \\
\text { típica }\end{array}$ & Moda & $\begin{array}{c}\text { Puntaje } \\
\text { Mínimo }\end{array}$ & $\begin{array}{c}\text { Puntaje } \\
\text { MÁximo }\end{array}$ \\
\hline Cohesión & 40.35 & 5.71 & 43 & 14 & 49
\end{tabular}

\begin{tabular}{lccccc}
\multicolumn{1}{c}{ Área } & Media & $\begin{array}{c}\text { Desviación } \\
\text { típıcA }\end{array}$ & Moda & $\begin{array}{c}\text { Puntaje } \\
\text { Mínimo }\end{array}$ & $\begin{array}{c}\text { Puntaje } \\
\text { MÁxımo }\end{array}$ \\
\hline Adaptabilidad & 26.93 & 5.28 & 27 & 17 & 42 \\
Tipo familia & 33.64 & 4.16 & 32 & 18 & 44
\end{tabular}

En cuanto a los tipos de estructura familiar, respecto a la cohesión $11 \%$ resultó no relacionada, mientras que $89 \%$ fue semirrelacionada; acerca de la adaptabilidad, $86 \%$ se clasificó como rígida y sólo 14\% fue estructurada (tabla 2).

Tabla 2.

Porcentaje de los diferentes tipos de estructura familiar encontrados en la muestra.

\begin{tabular}{cccccc} 
TIPO DE COHESIÓN & \multicolumn{2}{c}{ TIPO DE } & TiPO DE FAMILIA \\
\hline $\begin{array}{c}\text { No } \\
\text { relacionada }\end{array}$ & $\begin{array}{c}\text { Semi- } \\
\text { relacionada }\end{array}$ & Rígida & Estructurada & Extrema & $\begin{array}{c}\text { Rango } \\
\text { medio }\end{array}$ \\
$11 \%$ & $89 \%$ & $86 \%$ & $14 \%$ & $58 \%$ & $42 \%$
\end{tabular}

En el análisis de las correlaciones se identificó una asociación leve negativa significativa entre el nivel de cohesión y la escolaridad del paciente $(r=-0.27$; $p<0.05)$, lo que indica que el grado de unión emocional (o apego) percibido por los miembros de la familia es mayor cuando los pacientes tienen menos escolaridad; se encontró también una asociación moderada entre el nivel de adaptabilidad y las complicaciones $(r=-0.4 ; p<0.01)$, lo que indica que habrá menos posibilidades de adaptación familiar si se presenta un mayor número de complicaciones (hemorragias, artropatías, etcétera).

Tabla 3.

Correlación entre las principales variables sociodemográficas y las diferentes áreas del FACES III.

\begin{tabular}{|c|c|c|c|c|c|c|c|c|c|c|c|}
\hline & 1 & 2 & 3 & 4 & 5 & 6 & 7 & 8 & 9 & 10 & 11 \\
\hline $\begin{array}{l}\text { 1. Edad del } \\
\text { paciente }\end{array}$ & 1 & & & & & & & & & & \\
\hline 2. Severidad & 0.23 & 1 & & & & & & & & & \\
\hline $\begin{array}{l}\text { 3. Tipo de } \\
\text { tratamiento }\end{array}$ & 0.13 & $-0.37^{* *}$ & 1 & & & & & & & & \\
\hline $\begin{array}{l}4 . \\
\text { Hospitalizaciones }\end{array}$ & -0.14 & -0.25 & -0.017 & 1 & & & & & & & \\
\hline 5. Complicaciones & -0.07 & 0.04 & -0.04 & $0.48^{* *}$ & 1 & & & & & & \\
\hline $\begin{array}{l}\text { 6. Escolaridad del } \\
\text { paciente }\end{array}$ & $0.66^{* *}$ & 0.17 & 0.04 & 0.02 & 0.16 & 1 & & & & & \\
\hline 7. Edad del padre & $0.48^{*+}$ & 0.14 & 0.07 & -0.02 & -0.03 & $0.52^{* *}$ & 1 & & & & \\
\hline $\begin{array}{l}\text { 8. Escolaridad del } \\
\text { padre }\end{array}$ & 0.09 & $-0.34^{* *}$ & $0.35^{* *}$ & 0.09 & 0.05 & 0.14 & -0.07 & 1 & & & \\
\hline $\begin{array}{l}\text { 9. Nivel de } \\
\text { cohesión }\end{array}$ & -0.14 & -0.11 & 0.10 & -0.07 & -0.09 & $-0.27^{*}$ & -0.14 & -0.12 & 1 & & \\
\hline $\begin{array}{l}\text { 10. Nivel de } \\
\text { adaptabilidad }\end{array}$ & -0.09 & 0.13 & 0.02 & -0.07 & $0.4^{* *}$ & -0.01 & -0.08 & -0.20 & 0.14 & 1 & \\
\hline $\begin{array}{l}\text { 11. Tipos de } \\
\text { familia }\end{array}$ & 0.11 & 0.02 & 0.17 & 0.23 & 0.19 & 0.07 & 0.05 & -0.12 & $0.29^{*}$ & $0.5^{* *}$ & 1 \\
\hline \multicolumn{12}{|c|}{${ }^{*} \mathrm{p}<0.05 ;{ }^{* *} \mathrm{p}<0.01$. } \\
\hline
\end{tabular}


Por último se pueden observar dos correlaciones positivas entre los niveles de cohesión $(r=0.29 ; p<0.05)$ y los niveles de adaptabilidad $(r=0.5 ; p<0.01)$ con los tipos de familias, lo que indica la influencia de cada uno de estos constructos en la estructuración y funcionamiento familiar (tabla 3).

Para analizar si existían diferencias en la estructura familiar de acuerdo con algunas variables se aplicó la t de Student, obteniendo dos datos interesantes de comentar: el primero se refiere a que se encontraron diferencias significativas en los niveles de cohesión y de adaptabilidad entre la hemofilia A (HA) y la hemofilia B (HB) (tabla 4).

Tabla 4.

Diferencias entre la $H A$ y la $H B$.

\begin{tabular}{lccc}
\multicolumn{1}{c}{ Categoría } & T & $\begin{array}{c}\text { Grados DE } \\
\text { LIBERTAD }\end{array}$ & $\begin{array}{c}\text { Nivel DE } \\
\text { SIGNIFICANCIA }\end{array}$ \\
\hline $\begin{array}{l}\text { Nivel de cohesión } \\
\begin{array}{l}\text { Nivel de } \\
\text { adaptación }\end{array}\end{array}$ & -2.57 & 37 & 0.03 \\
$\begin{array}{l}\text { Tipo de familia } \\
\text { Sipifer }\end{array}$ & 0.09 & 37 & 0.004 \\
\end{tabular}

El segundo dato - y el más relevante para este estudiofueron diferencias en el nivel de adaptación, dependiendo si la familia asiste o no a tratamiento psicológico (tabla 5).

\section{Tabla 5.}

Diferencias entre asistir o no a tratamiento psicológico.

\begin{tabular}{lccc}
\multicolumn{1}{c}{ Categoría } & T & $\begin{array}{c}\text { Grados DE } \\
\text { LIBERTAD }\end{array}$ & $\begin{array}{c}\text { Nivel DE } \\
\text { SIGNIFICANCIA }\end{array}$ \\
\hline $\begin{array}{l}\text { Nivel de cohesión } \\
\begin{array}{l}\text { Nivel de } \\
\text { adaptación }\end{array}\end{array}$ & 1.24 & 37 & 0.21 \\
Tipo de familia & 1.21 & 37 & 0.03 \\
& 0.17 & 37 & 0.86
\end{tabular}

Finalmente, al comparar las diversas variables con la condición padres acompañantes no existieron diferencias significativas en ningún índice.

\section{CONCLUSIONES}

Efectuar estudios para analizar la estructura familiar de personas que viven un proceso de salud/enfermedad brinda elementos para observar los cambios que se producen al interior de estos grupos primarios y obtener herramientas para programar actividades de apoyo que les permitan superar y/o adaptarse mejor a dicha condición (Osorio y Graña, 2016).

En el presente estudio fue posible observar cómo familias que tienen un miembro diagnosticado con hemofi- lia y presentan variables sociodemográficas más o menos homogéneas (tratamiento adecuado, acceso a la Fundación y todos sus servicios médicos, atención psicológica etcétera), tienen estructuras familiares distintas, lo cual se refleja en la amplitud de sus respuestas en el cuestionario aplicado. Lo anterior fue reportado también por Kelley (2006) en un estudio que realizó con familias de varios países de Latinoamérica, donde encontró diferencias no sólo de un estado a otro, sino incluso a nivel local.

Al calcular los índices y clasificar a las familias de los participantes se obtuvo que respecto a la cohesión, la mayoría es del tipo semirrelacionada (o separada), lo que implica en cierto grado que sus integrantes son capaces de ser independientes $y$, al mismo tiempo, pueden estar relacionados con sus familias (Olson, Russell y Sprenkle, 1989; Schmidt, 2001). En cuanto al constructo de adaptabilidad -la cual se refiere al grado en que la familia es capaz de cambiar su estructura de poder, sus roles y reglas en respuesta al estrés-se encontró que un porcentaje alto de las familias se clasifican como rígidas, lo que implica un estilo familiar autocrático, en donde la capacidad de cambio es limitada. Ambas características - con pequeñas variantes - fueron encontradas en un estudio precedente, con familias mexicanas de pacientes con hemofilia (Osorio y Graña, 2016).

Respecto a las asociaciones encontradas se evidenció un mayor nivel de apego percibido por los miembros de la familia cuando los pacientes tienen menos escolaridad; esto puede indicar que (debido a la edad) los más jóvenes aún no hayan iniciado su instrucción o que los pacientes (debido al padecimiento) pasen mayor tiempo en casa y esto fomente dicha cohesión; lo anterior puede no ser necesariamente adecuado para el desarrollo integral del paciente (Osorio, 2016).

Asimismo se encontró una asociación moderada entre el nivel de adaptabilidad y las complicaciones, lo que indica que habrá menos posibilidades de adaptación familiar si se presenta un mayor número de complicaciones (hemorragias, artropatías, etcétera), debido a que el grupo familiar tendrá que modificar constantemente sus actividades y rutina para acompañar al paciente al hospital o para brindarle la atención necesaria en casa, datos que coinciden con lo indicado por Arranz, Costa, Bayés, Cancio, Magallón y Fernández (2000).

También se analizó el programa "Padres Acompañantes", variable en la cual no se obtuvieron datos estadísticamente significativos; sin embargo, en la práctica u observación directa se muestra un impacto cualitativo que trae aparejada una mejora en el bienestar general de las familias acompañadas, siendo reportado por otros autores en eventos académicos y de divulgación (Graña y Rodríguez, 
2000; Graña, 2010, 2014). Esta situación impulsa a apoyar y a difundir con mayor ímpetu el programa, ya que se tiene la convicción de su utilidad clínica.

Uno de los datos más sobresalientes es haber encontrado diferencias en las estructuras familiares de pacientes con $\mathrm{HA}$ y $\mathrm{HB}$, de lo que se desprende que el tipo de organización familiar se ve afectada de manera desigual, aunque médicamente las manifestaciones clínicas son iguales en ambas hemofilias.

Otro resultado interesante es la diferencia significativa que existe en la adaptabilidad de la familia, dependiendo de si asisten o no a terapia psicológica, ya que el dato muestra la importancia de asistir a este servicio, que enseña y otorga herramientas que facilitan la adaptación a la condición de la familia para afrontar la enfermedad crónica.

No obstante a lo anterior, como punto de reflexión las autoras se plantean la necesidad de investigar si las familias que no asisten a terapia son — por sí mismasmenos adaptadas, y eso determina que no acudan a consulta psicológica; además de agregar al análisis variables como tiempo de trayecto a la Fundación y días/ horarios del servicio. Asimismo, para futuras investigaciones se pretende efectuar entrevistas que complementen la información recabada con los instrumentos.

Con base en estos resultados, se proyectarán diversos programas que difundan la importancia de asistir al servicio psicológico y facilitar a las familias que no estén asistiendo al servicio, información acerca de la relevancia de este tipo de apoyo. Se seguirá fomentando — como ya se mencionó- el programa "Padres Acompañantes" para que tenga un impacto mayor en la población de la Fundación.

\section{REFERENCIAS}

Arranz, P., Costa, M., Bayés, R., Cancio, H., Magallón, M. \& Fernández, F. (2000). El apoyo emocional en hemofilia. Madrid: Industrias Gráficas Sprint.

Balcells, M. \& Ribalta, T. (2000). Psychological examinations for the early detection of madurative dysfunctions in hemophiliac children: preliminary results. Haemophilia, 6 (suppl. 4): 413.

Benoit de García, H. (2008). Sharing a hard life: how hemophilia affects the family. A Mother's perspective. Haemophilia, 14 (suppl. 2): 129.

Consenso de Médicos Especialistas en Hemofilia de la República Argentina (2015). Guía para el manejo de la Hemofilia Congénita. Argentina: Fundación de la Hemofilia.

Goldstein, G. (2008). Siblings interaction in families with hemophilia. Haemophilia, 14 (suppl. 2): 129.

Goldstein, G., Golan, G., Kenet, G. \& Martinowitz, U. (2000). From the beginning: Group therapy with parents of babies with hemophilia. Haemophilia, 6 (suppl. 4): 417.

Graña, S. \& Rodríguez, O. M. (2000). Experience with
Accompanying Parents. Haemophilia, 6 (suppl. 4): 418.

Graña, S. (2010). Initial diagnosis: strategies to promote acceptance. Haemophilia, 16 (suppl. 4): 128.

Graña, S. (2014). Growing up with Hemophilia: Children's Perspective. Haemophilia, 20 (suppl. 3): 158.

Hashim, A. (2008). Sharing a hard life: how hemophilia affects the family. A Father's perspective, Haemophilia, 14 (suppl. 2): 129.

Herrick, E., Nussbaum, R., Holtzman, N.A. \& Wissow, L. (2004). Asking Fathers: a study of psychosocial adaptation. Haemophilia, 10 (5): 582-589.

Kelley, L. (2006). Criando un niño con hemofilia en América Latina. USA: Baxter Bioscience.

O'Mahony, B. (2002). What we must do to improve hemophilia care around the world. HemAware, 7 (2). 46-51.

Olson, D. H. (2000). Circumplex model of Marital and Family Systems, Journal of Family Therapy, 22: 144-167.

Olson, D. H., Portner, J. \& Lavee, Y. (1985). Manual de la Escala de Cohesión y Adaptabilidad Familiar (FACES III Manual). Minneapolis: Life Innovation.

Olson, D. H., Russell, C. \& Sprenkle, D. H. (1989). Circumplex Model of Marital and Family Systems II: Empirical studies and clinical intervention. Advances in Family Intervention, Assessment and Theory, 1: 129-176.

Olson, D. H., Sprenkle, D. H. \& Russell, C. S. (1979). Circumplex model of marital and family systems, I: Cohesion and adaptability dimensions, family types, and clinical applications. Family Process, 18: 3-28.

Osorio G. M. (2016). Repercusiones biopsicosociales en los padres que tienen un hijo con hemofilia. Revista de Investigación en Psicología, 19 (2): 111-121.

Osorio, G. M, y Graña, S. (2016). Descripción de la estructura familiar de pacientes con hemofilia. Comparación ArgentinaMéxico. Revista Costarricense de Psicología, 35 (1), 1-15.

Ponce, R. E., Gómez, C. F., Terán, T. M., Irigoyen, C. A. \& Landgrave, I. S. (2002). Validez de constructo del cuestionario FACES III en español (México). Aten Primaria, 30 (10): 624-630.

Ponce, R. E., Gómez, C. F., Irigoyen, C. A., Terán, T. M., Landgrave, I. S., Hernández, G. A. et al. (1999). Análisis de la confiabilidad de FACES III (versión en español). Aten Primaria, 23: 479-84.

Pruthi, R. K. (2005). Hemophilia: a practical approach to genetic testing. Mayo Clin Proc, 80, 1485-99.

Schmidt, V. (2001). Recursos para el afrontamiento de eventos vitales estresantes en familias de drogodependientes. RIDEP, 11 (1): 75-92.

Schmidt, V. (2003). Estrés en familias de drogodependientes. Tesis doctoral. Buenos Aires: Facultad de Psicología, U. B. A.

Schmidt, V. Barreyro, J. P. \& Maglio, A. L. (2009). Escala de evaluación del funcionamiento familiar FACES III: ¿Modelo de dos o tres factores? Escritos de Psicología, 3 (2): 30-36.

Shaughnessy, J. J., Zechmeister, E. B., \& Zechmeister, J. S.(2007). Métodos de investigación en psicología. México: Mc Graw Hill.

Spilsbury, M. (2004). Models for psychosocial services in the developed and developing world. Haemophilia, 10 (suppl. 4). 25-29.

Winnicott, D. (1979). Objetos y fenómenos transicionales: estudio de la primera posesión "no yo". En: Escritos de pediatría y psicoanálisis: 1931/1956. Barcelona: Laia.

Winnicott, D. (1982). Realidad y juego. Buenos Aires: Gedisa. 


\section{Meta-Análisis del Artículo}


Revista Digital Internacional de Psicología y Ciencia Social | Vol. 3 | Núm. 2 | Juilio-Diciembre 2017| ISSN 2448-8119 e-ISSN $2448-8119$

\section{Dimensión Cuantitativa}

\section{Perfil de Evaluación entre pares}
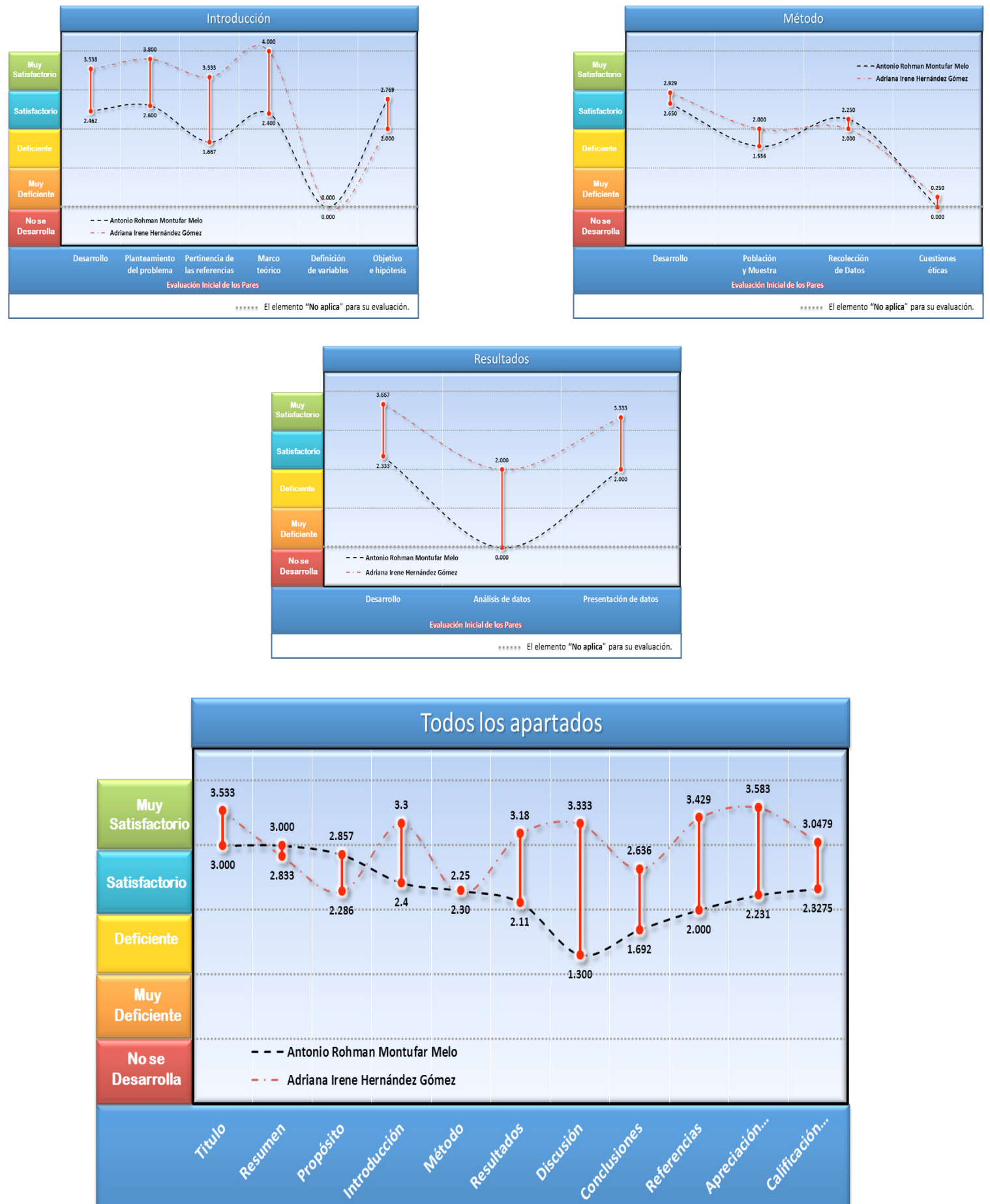


\section{Índice de Concordancia}

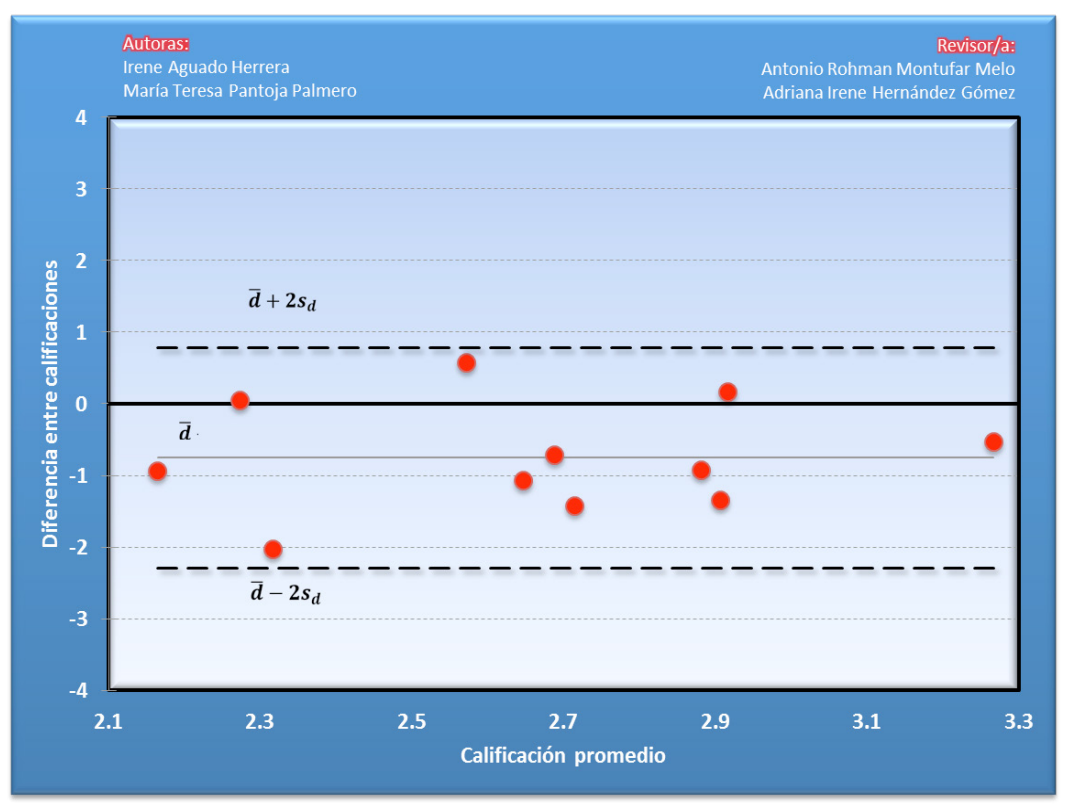

Índice de Acuerdo
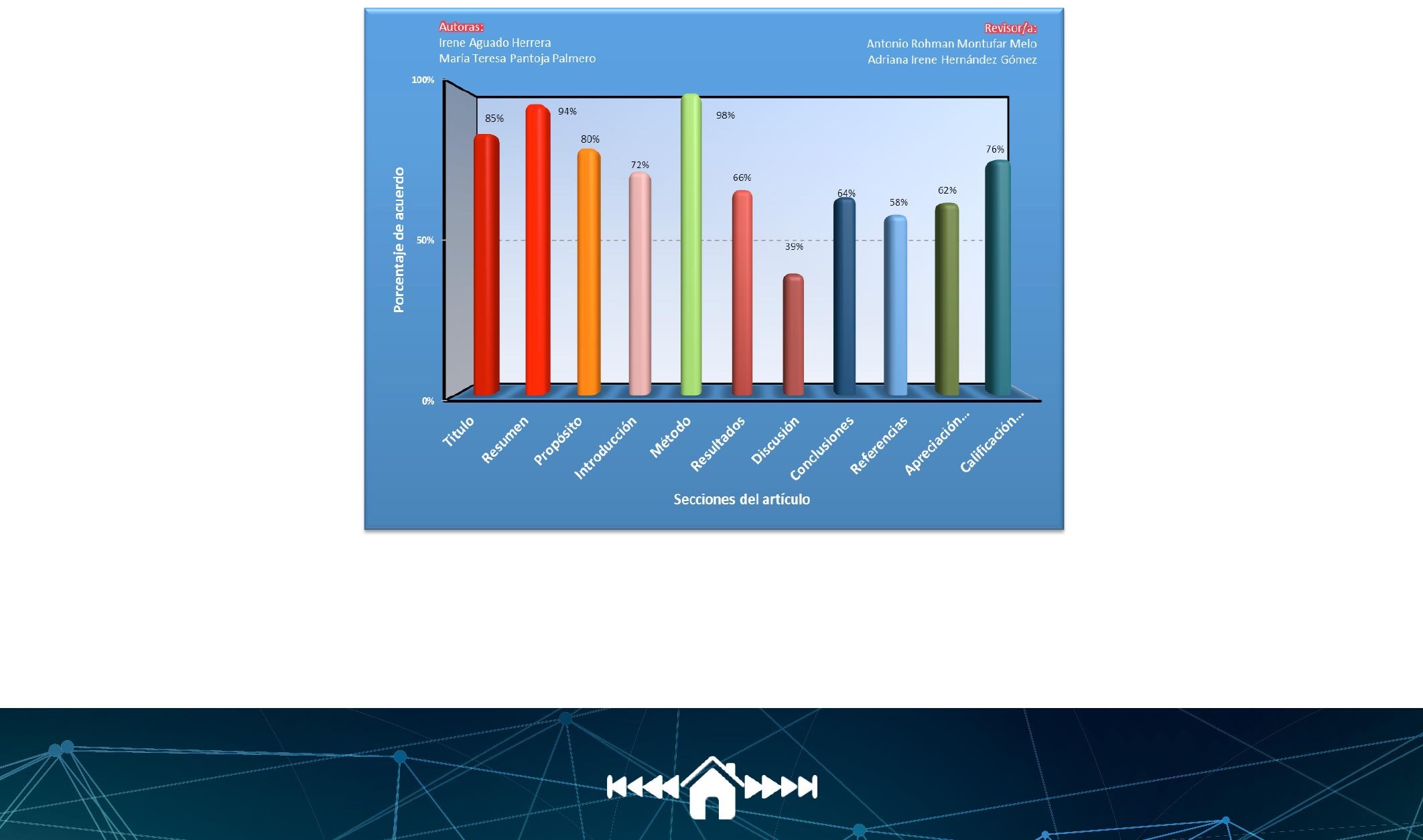


\begin{tabular}{|c|c|}
\hline Revisor 1 & Revisor 2 \\
\hline \multicolumn{2}{|c|}{ Resultados } \\
\hline $\begin{array}{l}\text { Para facilitar la lectura, conviene presentar previamen- } \\
\text { te la descripción de las categorías Cohesión y Adaptabi- } \\
\text { lidad en el apartado metodológico; lo mismo vale para } \\
\text { los tipos de estructura familiar: relacionada, semi-re- } \\
\text { lacionada y tipo de familia. Se sugiere aclarar por qué } \\
\text { los autores tomaron la decisión de usar el estadígrafo r } \\
\text { de correlación, ya que al ser una muestra tan pequeña } \\
\text { se pierde la fuerza estadística; quizá sea mejor utilizar } \\
\text { la ji-cuadrada en cada instrumento. Se fuerza la signi- } \\
\text { ficancia estadística usando un coeficiente que no sería } \\
\text { conveniente aplicar ya que la muestra es muy chica. }\end{array}$ & No hay comentarios. \\
\hline \multicolumn{2}{|c|}{ Discusión } \\
\hline $\begin{array}{l}\text { Antes de las conclusiones, sería muy apreciable que los } \\
\text { autores agregasen un apartado de DISCUSION en el } \\
\text { que comparan sus resultados con otros autores que han } \\
\text { trabajado en el mismo tema, haciendo la salvedad de la } \\
\text { limitación de por el tipo de muestreo y por el tamaño } \\
\text { de la muestra. }\end{array}$ & $\begin{array}{l}\text { Quiza analizar estudios en nuestro pais y las caracteristicas de las } \\
\text { familias mexicana, aportarian nuevas vertientes en su trabajo. }\end{array}$ \\
\hline \multicolumn{2}{|c|}{ Conclusiones } \\
\hline $\begin{array}{l}\text { Se sugiere tener cautela con la aseveración "la diferen- } \\
\text { cia significativa que existe en la adaptabilidad de la fa- } \\
\text { milia dependiendo de si asisten o no a terapia psicoló- } \\
\text { gica" teniendo en cuenta los estadígrafos utilizados. }\end{array}$ & $\begin{array}{l}\text { Me parece que seria muy interesante proponer mas inves- } \\
\text { tigaciones con familias con otras enfermedades cronicas, } \\
\text { de otros paises, comparando mas variables, etc. Les falta- } \\
\text { ria agregar un poco mas de estas ideas. }\end{array}$ \\
\hline \multicolumn{2}{|c|}{ Referencias } \\
\hline $\begin{array}{l}\text { Revisar el Manual de Estilo de la American Psychologi- } \\
\text { cal Association (APA) para referenciar adecuadamente } \\
\text { las fuentes consultadas. }\end{array}$ & $\begin{array}{l}\text { Aunque mucha informacion tiene entre } 10 \text { y } 15 \text { años de } \\
\text { antiguedad, o incluso mas, son necesarias de retomar y no } \\
\text { creo que demerite el escrito. }\end{array}$ \\
\hline
\end{tabular}


